\title{
Discrete inverse Sumudu transform application to Whittaker equation and Zettl equation
}

\author{
Rathinavel Silambarasan ${ }^{1}$, Kottakkaran Sooppy Nisar $^{2}$ and Fethi Bin Muhammad Belgacem ${ }^{3, \star}$ \\ ${ }^{1}$ Department of Information Technology, SITE, VIT University, Vellore, Tamilnadu, India. Email : silambu_vel@ yahoo.co.in \\ 2 Department of Mathematics, College of Arts and Science, Prince Sattam bin Abdulaziz University, Wadi Al dawaser, \\ Riyadh region 11991, Alkharj, Kingdom of Saudi Arabia. Email : ksnisar1@gmail.com \\ ${ }^{3}$ Department of Mathematics, Faculty of Basic Education, PAAET, Al-Ardhiya, Kuwait. Email : fbmbelgacem@ gmail.com \\ * Corresponding Author.
}

\begin{abstract}
Inverse Sumudu transform multiple shifting properties are used to design methodology for solving ordinary differential equations. Then algorithm applied to solve Whittaker and Zettl equations to get their new exact solutions and profiles which shown through Maple complex graphicals. Table of inverse Sumudu transforms for elementary functions given for supporting the differential equations solving using inverse Sumudu transform.
\end{abstract}

Keywords : Discrete inverse Sumudu transform, Whittaker equation, Zettl equation, Gauss hypergeometric series and modified Struve function.

\section{Introduction}

Inverse Sumudu transform applied for Bessel's differential equation of order zero and solved with power series solution in [5]. New definition for Sumudu transform of trigonometric function by integrating the function followed by Sumudu inverting the new definition, trigonometric functions are expressed as new infinite series where the series coefficients are obtained by integrating the function and evaluating at the origin, with examples and table of such series of set of trigonometric functions given in [6]. Jacobi elliptic functions with two modulus denoting bimodular Jacobi elliptic functions are Sumudu transformed to expand as associated continued fractions, from which Hankel determinants are derived from those coefficients, all for higher powers and modular transformation studied by Belgacem and Silamabarasan . For more about Sumudu transform and its applications one may be referred to $[3,9,12,13]$. Integral equation definition of Sumudu transform for the function $f(t)$ in $A=\left\{f(t)\left|\exists M, \tau_{1}, \tau_{2}>0,\right| f(t) \mid<M e^{\frac{|t|}{\tau_{j}}}\right.$,if $\left.t \in(-1)^{j} \times[0, \infty)\right\}$ takes,

$$
\mathbb{S}[f(t)](u)=F(u)=\int_{0}^{\infty} e^{-t} f(u t) d x=1 / u \int_{0}^{\infty} e^{-t / u} f(t) d t ; u \in\left(-\tau_{1}, \tau_{2}\right) .
$$

While for the Discrete equation definition Sumudu of $f(t)$ in $A$ is,

$$
\mathbb{S}[f(t)](u)=F(u)=\sum_{n=0}^{\infty} f^{(n)}(0) u^{n} .
$$

where $f(t)=\sum_{n=0}^{\infty} \frac{f^{(n)}(0) t^{n}}{n !}$ is the series (Maclaurin) expansion of function. Thus the variable $t$ Sumudu transforms to variable $u$. While on the other hand let the variable $t$ inverse Sumudu transform to variable $w$. Therefore Discrete inverse Sumudu transform (DIST) of $f(t)$ in $A$ is,

$$
\mathbb{S}^{-1}[f(t)](w)=F_{-1}(w)=\sum_{n=0}^{\infty} \frac{f^{(n)}(0) w^{n}}{(n !)^{2}} .
$$


Following in the same way inverse Sumudu transform of $\cos (\alpha t)$ is given by $\operatorname{ber}_{0}(2 \sqrt{a w})$. For the set of functions taken from [10], table constituting functions and their inverse Sumudu transform given in Table 3 where certain function definitions given in Table 4.

In this communication DIST is applied to Whittaker equation and Zettl equation to obtain their solution through known inverse Sumudu transform properties. This work is organised as inverse Sumudu transform properties and DIST methodology description in Section 2, in Sections 3 and 4 respective DIST application to Whittaker equation and Zettl equation followed by Conclusion at Section 5.

\section{Inverse Sumudu transform properties}

Theorem 1. Let $F_{-1}(w)$ be the inverse Sumudu transform of $f(t)$.

$$
\begin{aligned}
\mathbb{S}^{-1}\left[t^{n} \frac{d^{n} f(t)}{d t^{n}}\right] & =w^{n} \frac{d^{n} F_{-1}(w)}{d w^{n}} . \\
\mathbb{S}^{-1}\left[t^{n} \frac{d^{n} t^{n} f(t)}{d t^{n}}\right] & =w^{n} F_{-1}(w) . \\
\mathbb{S}^{-1}\left[t^{n} f(t)\right] & =\underbrace{\int_{0}^{w} \cdots \int_{0}^{w}}_{n-\text { times }} F_{-1}(\eta)(d \eta)^{n} . \\
\mathbb{S}^{-1}\left[\frac{f(t)}{t^{n}}\right] & =\frac{d^{n} F_{-1}(w)}{d w^{n}} .
\end{aligned}
$$

Proof. The proof follows from multiple shifting properties of Sumudu transform [5].

DIST steps to solve ordinary differential equations with polynomial coeffecients is given in Algorithm 1.

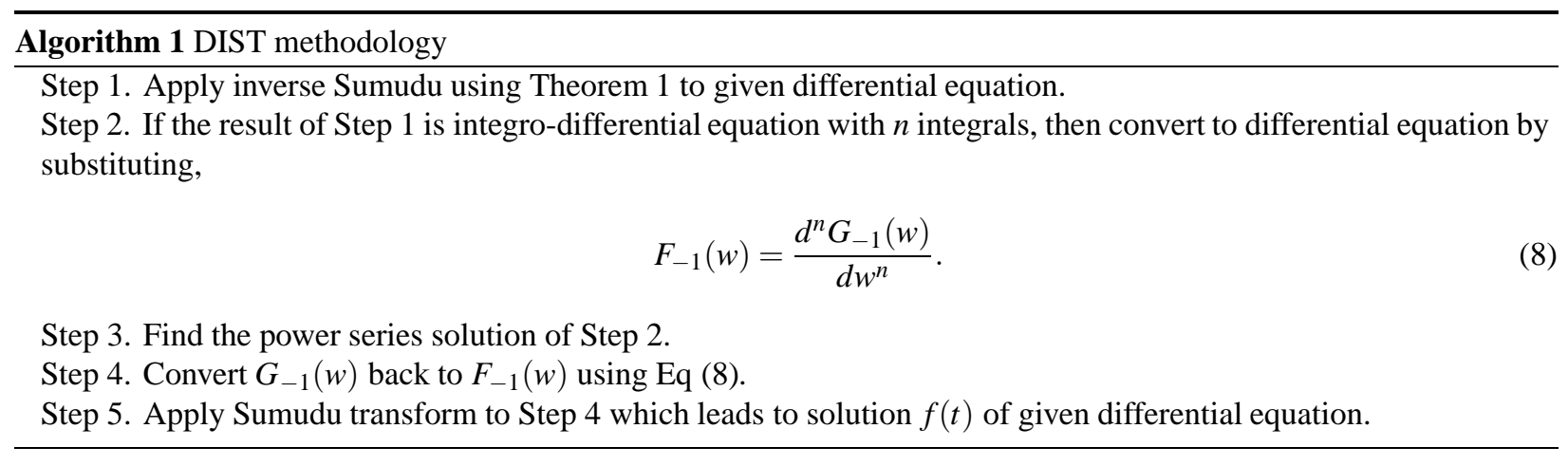

\section{DIST application to Whittaker equation}

Whittaker equation end point boundary classification in Lebesgue space given in $[4,8]$ while generalized solutions in [1]. Whittaker differential equation is given by (pp 299, [4]).

$$
-f^{\prime \prime}(t)+\left[\frac{1}{4}+\frac{k^{2}-1}{t^{2}}\right] f(t)=\frac{\lambda f(t)}{t} \forall t \in(0,+\infty) .
$$

with

$$
k \in[1, \infty), \lambda_{n}=n+\left(\frac{k+1}{2}\right) ; n=0,1,2, \cdots
$$


Inverse Sumudu application of (9) gives following integro-differential equation.

$$
-w^{2} F_{-1}^{\prime \prime}(w)+\frac{1}{4} \int_{0}^{w} \int_{0}^{w} F_{-1}(\eta)(d \eta)^{2}+\left(k^{2}-1\right) F_{-1}(w)=\lambda \int_{0}^{w} F_{-1}(\eta) d \eta .
$$

Removing the integrals of Eq (11) gives,

$$
-w^{2} G_{-1}^{(4)}(w)+\frac{1}{4} G_{-1}(w)+\left(k^{2}-1\right) G_{-1}^{(2)}(w)=\lambda G_{-1}^{(1)}(w) .
$$

The power series solutions of Eq (12) with $k=1$ and $\lambda=0$ through 6 are given Table 1 .

Table 1: Power series of solution of Eq (12) with $k=1$ and for different values of $\lambda$

\begin{tabular}{|c|c|c|}
\hline S.No & $\lambda$ & $G_{-1}(w)$ \\
\hline 1 & 0 & $16 \sum_{n=1}^{\infty} \frac{2^{4 n}(2 n-1) n w^{2 n}}{64^{n} \Gamma(2 n+1)^{2}}+12 \sum_{n=1}^{\infty} \frac{2^{4 n} w^{2 n+1}}{64^{n}(2 n+1) \Gamma(2 n)^{2}}$. \\
3 & 1 & $-48 \sum_{n=3}^{\infty} \frac{(-1)^{n}(n-2)(n-1) n w^{n}}{2^{n} \Gamma(n+1)^{2}}$. \\
4 & 3 & $8 \sum_{n=3}^{\infty} \frac{(-1)^{n}(n-2)(n-1) n(-2 n+4+i \sqrt{2})(2 n-4+i \sqrt{2}) w^{n}}{2^{n} \Gamma(n+1)^{2}}$ \\
5 & 4 & $16 \sum_{n=3}^{\infty} \frac{(-1)^{n}(n-2)^{2}(n-1) n(-n+2+i \sqrt{2})(n-2+i \sqrt{2}) w^{n}}{2^{n} \Gamma(n+1)^{2}(n+1)^{2}}$. \\
6 & 5 & $\sum_{n=3}^{\infty} \prod_{k=3}^{n-1} \frac{-\left(2 k^{4}-8 k^{3}+22 k^{2}-28 k+15\right) w^{n}}{2(k-2)(k+1)\left(2 k^{4}-16 k^{3}+58 k^{2}-104 k+75\right)}$. \\
7 & 6 & $\sum_{n=3}^{\infty} \prod_{k=3}^{n-1} \frac{-\left(2 k^{4}-8 k^{3}+32 k^{2}-48 k+45\right)(k-1) w^{n}}{2(k-2)^{2}(k+1)\left(2 k^{4}-16 k^{3}+68 k^{2}-144 k+135\right)}$. \\
\hline
\end{tabular}

Next using Step 4 and Step 5 of Algorithm 1, converting column 3 of Table 1 back to $F_{-1}(w)$ and applying Sumudu transform gives the solution $f(t)$ of Eq (9) which is given in Table 2.

Table 2: Solution $f(t)$ of $\mathbf{E q}(9)$ with $k=1$ and for different values of $\lambda$

\begin{tabular}{|c|c|c|}
\hline S.No & $\lambda$ & $f(t)$ \\
\hline 1 & 0 & $2 \cosh \left(\frac{t}{2}\right)+12 \sinh \left(\frac{t}{2}\right)$ \\
& & $6 t \exp \left(-\frac{1}{2} t\right)$. \\
\hline
\end{tabular}

Continued on next page 
Table 2 - Continued from previous page

\begin{tabular}{|c|c|c|}
\hline S.No & $\lambda$ & $f(t)$ \\
\hline 3 & 2 & $-3 t(t-2) \exp \left(-\frac{1}{2} t\right)$. \\
4 & 3 & $-\frac{1}{6(i \sqrt{2}-1)}\left[(-2+i \sqrt{2})(-4+i \sqrt{2})\left(t^{2}-6 t+6\right) t \exp \left(-\frac{1}{2} t\right)\right]$. \\
5 & 4 & $-\frac{t}{4}\left[t^{3}-12 t^{2}+36 t-24\right] \exp \left(-\frac{1}{2} t\right)$. \\
7 & 5 & $\frac{t}{20}\left[t^{4}-20 t^{3}+120 t^{2}-240 t+120\right] \exp \left(-\frac{1}{2} t\right)$. \\
& & $-\frac{t}{120}\left[t^{5}-30 t^{4}+300 t^{3}-1200 t^{2}+1800 t-720\right] \exp \left(-\frac{1}{2} t\right)$. \\
\hline
\end{tabular}

Graphical behaviour of solutions $f(t)$ of Eq (9) for different values of $\lambda$ shown in Figure 1.

\subsection{Interpretation of results}

Following observation made from the study of Whittaker equation with DIST.

- All the solution $f(t)$ in Table 2 are new exact solutions which are verified using Maple and appearing for first time.

- From Table 2 in the solution $f(t)$, for $\lambda=1$ order of the polynomial is one, for $\lambda=2$ order of the polynomial is two. Thus for general $\lambda$ polynomial of order $\lambda$, hence solution $f(t)$ is $O(\lambda) \exp \left(-\frac{1}{2} t\right)$.

- For $\lambda=3$ gives the exact complex solution.

- For $k>1$ in Whittaker equation, DIST gives approximate (or) truncated power series solution.

\section{DIST application to Zettl equation}

Zettl differential equation is given by [4] which is related to Fourier equation [2,11].

$$
-\left[\sqrt{t} f^{\prime}(t)\right]^{\prime}=\frac{\lambda f(t)}{\sqrt{t}} .
$$

Inverse Sumudu transform of Eq (13) leads to,

$$
-\frac{w}{2} F_{-1}^{\prime}(w)-w^{2} F_{-1}^{\prime \prime}(w)=\lambda \int_{0}^{w} F_{-1}(\eta) d \eta
$$

Converting Eq (14),

$$
-\frac{w}{2} G_{-1}^{\prime \prime}(w)-w^{2} G_{-1}^{(3)}(w)=\lambda G_{-1}(w) .
$$

The power series solution of Eq (15) is, 


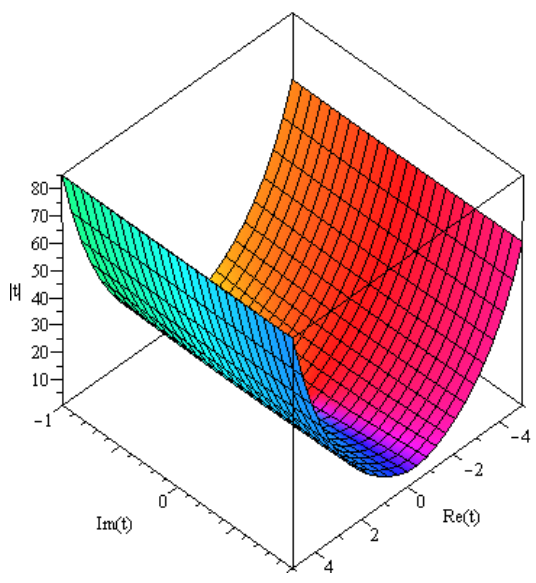

(a) $\lambda=0$

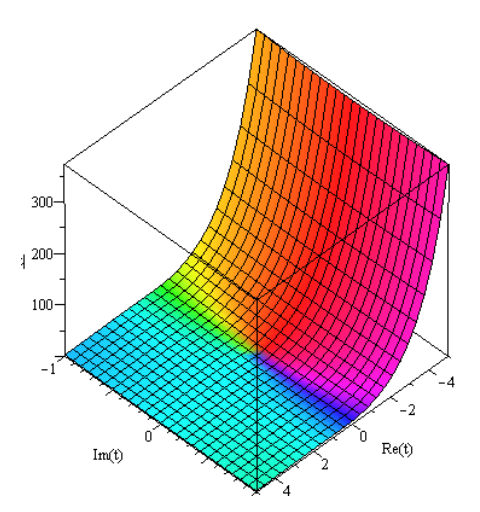

(b) $\lambda=1$

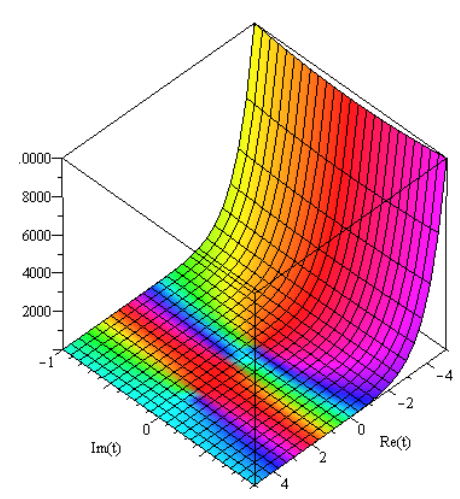

(e) $\lambda=4$

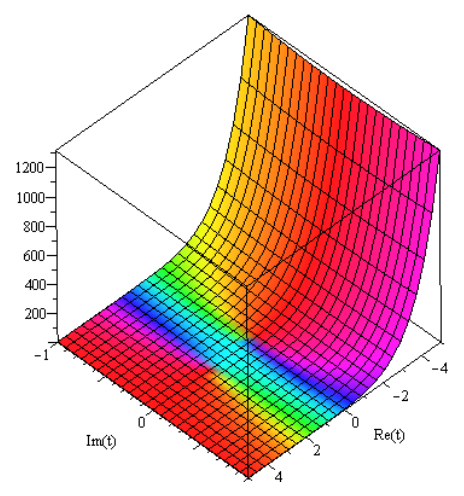

(c) $\lambda=2$

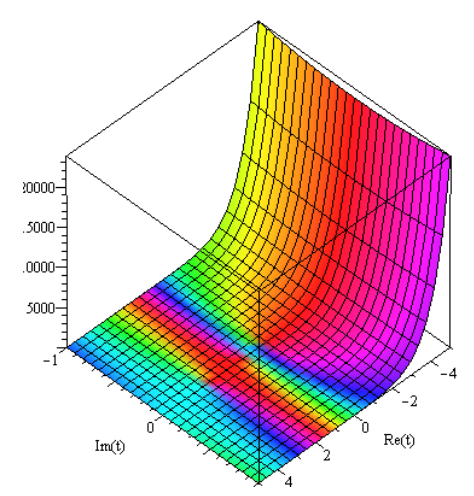

(f) $\lambda=5$

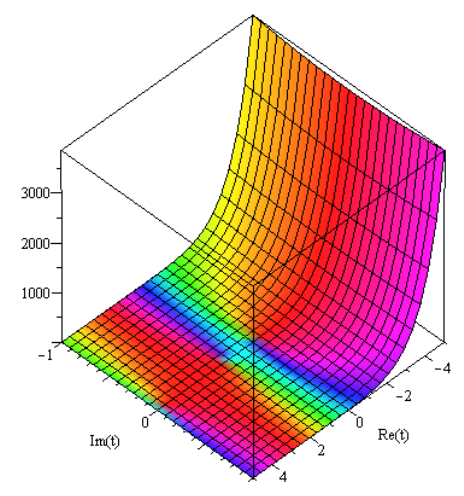

(d) $\lambda=3$

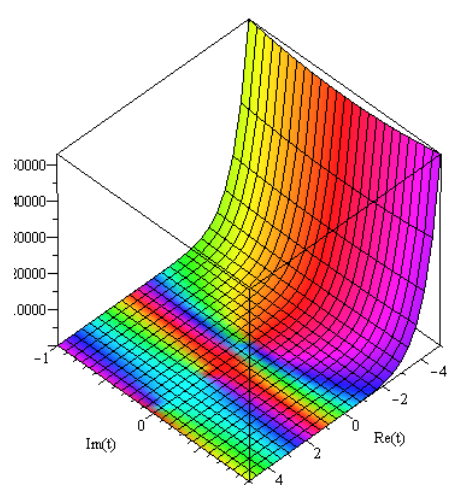

(g) $\lambda=6$

Figure 1: 3D complex plots of solution $f(t)$ of Eq (9) with $k=1$ and different values of $\lambda$ corresponding to column 3 of Table 2. 


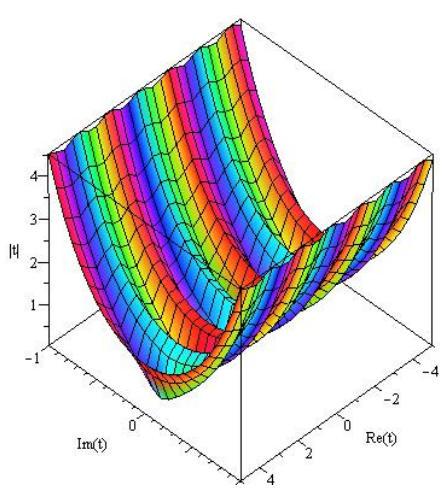

(a) $\lambda=1$

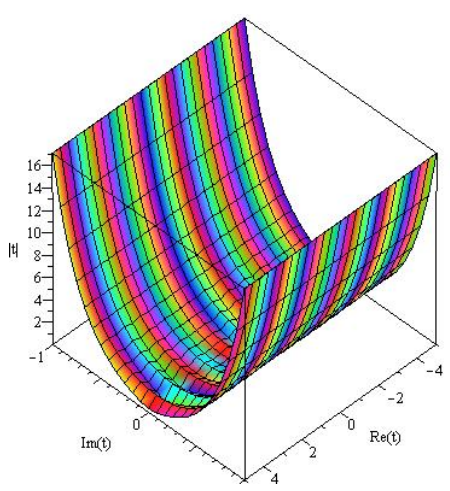

(c) $\lambda=3$

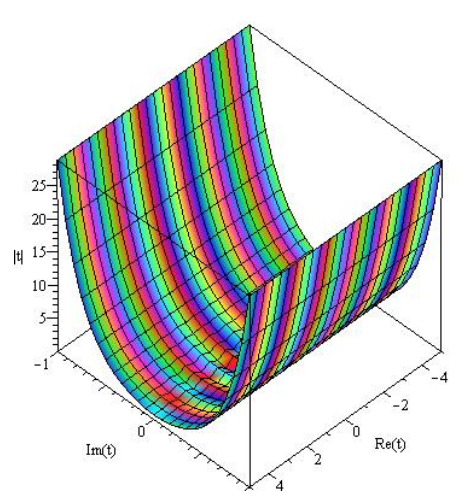

(d) $\lambda=4$

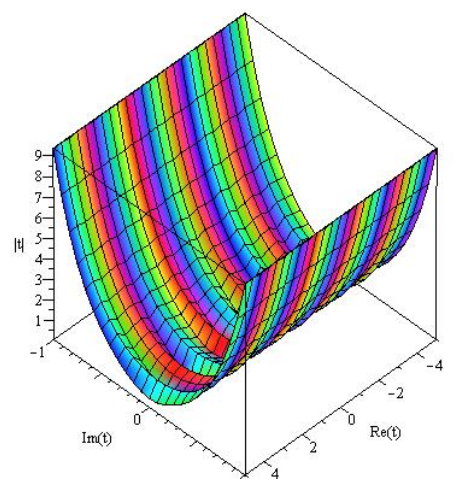

(b) $\lambda=2$

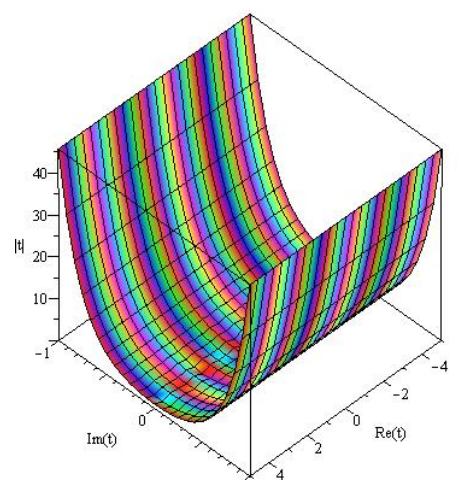

(e) $\lambda=5$

Figure 2: 3D complex plots of solution $f(t)$ of $\mathbf{E q}$ (13) for different values of $\lambda$.

$$
G_{-1}(w)=-\frac{\sqrt{\pi}}{\lambda} \sum_{n=1}^{\infty} \frac{(-\lambda)^{n} w^{n}}{n \Gamma(n)^{2} \Gamma\left(-\frac{1}{2}+n\right)}+\frac{3 \pi w^{3 / 2}}{16} \sum_{n=0}^{\infty} \frac{(-\lambda)^{n}(2 n+3) w^{n}}{\Gamma\left(n+\frac{5}{2}\right) \Gamma(n+1)} .
$$

Changing back to $F_{-1}(w)$ and Sumudu transform application gives the solution of Eq (13).

$$
f(t)=\cos (2 \sqrt{\lambda} t)+\frac{3}{8} \sqrt{\frac{\pi}{\lambda}} \sin (2 \sqrt{\lambda} t)
$$

\subsection{Interpretation of results}

Following observations made from Zettl equation study through DIST.

- Eq (17) is new exact solution of Zettl equation (13), verified using Maple.

- As the $\lambda$ value increase solution $f(t)$ forms the cusp shape as can be seen in Figure 2. 
Table 3: Inverse Sumudu transform of elementary functions

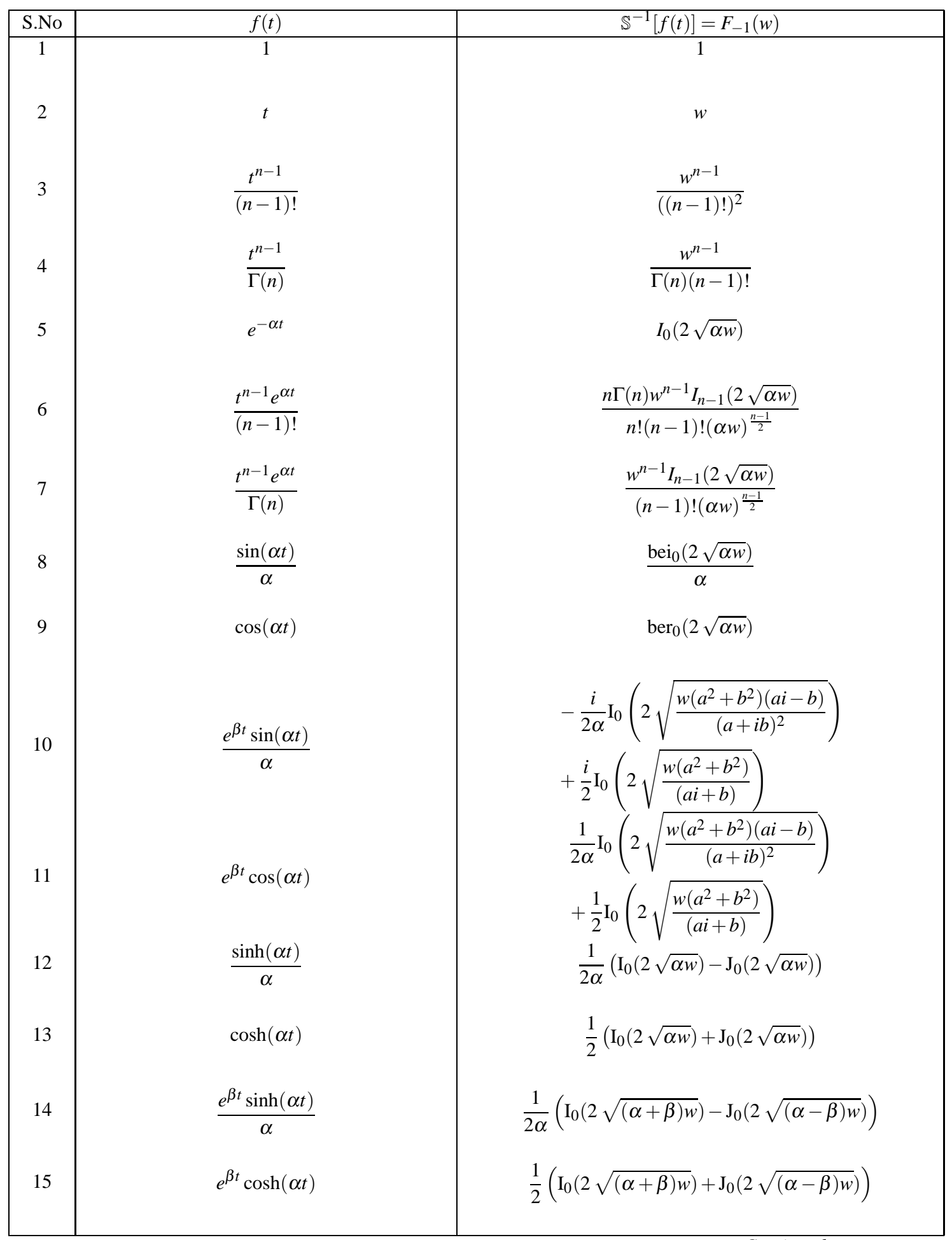

Continued on next page 
Table 3-Continued from previous page

\begin{tabular}{|c|c|c|}
\hline S.No & $f(t)$ & $\mathbb{S}^{-1}[f(t)]=F_{-1}(w)$ \\
\hline 16 & $\frac{e^{\beta t}-e^{\alpha t}}{\beta-\alpha}$ & $\frac{1}{\alpha-\beta}\left(\mathrm{I}_{0}(2 \sqrt{\alpha w})-\mathrm{I}_{0}(2 \sqrt{\beta w})\right)$ \\
\hline 17 & $\frac{\beta e^{\beta t}-\alpha e^{\alpha t}}{\beta-\alpha}$ & $\frac{1}{\alpha-\beta}\left(\alpha \mathrm{I}_{0}(2 \sqrt{\alpha w})-\beta \mathrm{I}_{0}(2 \sqrt{\beta w})\right)$ \\
\hline 18 & $\frac{\sin \alpha t-\alpha t \cos \alpha t}{2 \alpha^{3}}$ & $\begin{array}{c}\frac{1}{4 \alpha^{\frac{7}{2}} \sqrt{w}}\left(\sqrt{2} \alpha w\left(\operatorname{ber}_{1}(2 \sqrt{\alpha w})-\operatorname{bei}_{1}(2 \sqrt{\alpha w})\right)\right. \\
\left.+2 \sqrt{\alpha w} \operatorname{bei}_{0}(2 \sqrt{\alpha w})\right)\end{array}$ \\
\hline 19 & $\frac{t \sin (\alpha t)}{2 \alpha}$ & $-\frac{1}{4 \alpha^{\frac{3}{2}}}\left(\sqrt{2 w} \operatorname{ber}_{1}(2 \sqrt{a w})+\operatorname{bei}_{1}(2 \sqrt{a w})\right)$ \\
\hline 20 & $\frac{\sin \alpha t+\alpha t \cos \alpha t}{2 \alpha}$ & ${ }_{1} F_{4}\left(2 ; 1,1, \frac{3}{2}, \frac{3}{2} ;-\frac{(\alpha w)^{2}}{16}\right)$ \\
\hline 21 & $\cos \alpha t-\frac{\alpha t}{2} \sin \alpha t$ & ${ }_{1} F 4\left(2 ; \frac{1}{2}, \frac{1}{2}, 1,1 ;-\frac{(\alpha w)^{2}}{16}\right)$ \\
\hline 22 & $t \cos (\alpha t)$ & $-\frac{1}{2 \sqrt{\alpha}}\left(\sqrt{2 w} \operatorname{ber}_{1}(2 \sqrt{\alpha w})-\operatorname{bei}_{1}(2 \sqrt{\alpha w})\right)$ \\
\hline 23 & $\frac{\alpha t \cosh \alpha t-\sinh \alpha t}{2 \alpha^{3}}$ & $\begin{aligned}-\frac{1}{4 \alpha^{\frac{7}{2}} \sqrt{w}}( & \sqrt{\alpha w}\left(\mathrm{I}_{0}(2 \sqrt{\alpha w})-\mathrm{J}_{0}(2 \sqrt{\alpha w})\right) \\
& \left.-\alpha w\left(\mathrm{I}_{1}(2 \sqrt{\alpha w})+\mathrm{J}_{1}(2 \sqrt{\alpha w})\right)\right)\end{aligned}$ \\
\hline 24 & $\frac{t \sinh (\alpha t)}{2 \alpha}$ & $\frac{\sqrt{w}}{4 \alpha^{\frac{3}{2}}}\left(\mathrm{I}_{1}(2 \sqrt{\alpha w})-\mathrm{J}_{1}(2 \sqrt{\alpha w})\right)$ \\
\hline 25 & $\frac{\sinh \alpha t+\alpha t \cosh \alpha t}{2 \alpha}$ & $\begin{aligned} \frac{1}{4 \alpha^{\frac{3}{2}} \sqrt{w}} & \left(\sqrt{\alpha w}\left(\mathrm{I}_{0}(2 \sqrt{\alpha w})-\mathrm{J}_{0}(2 \sqrt{\alpha w})\right)\right. \\
& \left.+\alpha w\left(\mathrm{I}_{1}(2 \sqrt{\alpha w})+\mathrm{J}_{1}(2 \sqrt{\alpha w})\right)\right)\end{aligned}$ \\
\hline 26 & $\cosh \alpha t+\frac{\alpha t}{2} \sinh \alpha t$ & $\begin{array}{r}\frac{1}{4 \sqrt{\alpha w}}\left(2 \sqrt{\alpha w}\left(\mathrm{I}_{0}(2 \sqrt{\alpha w})+\mathrm{J}_{0}(2 \sqrt{\alpha w})\right)\right. \\
\left.+\alpha w\left(\mathrm{I}_{1}(2 \sqrt{\alpha w})-\mathrm{J}_{1}(2 \sqrt{\alpha w})\right)\right)\end{array}$ \\
\hline 27 & $t \cosh (\alpha t)$ & $\sqrt{\frac{w}{2 \alpha}}\left(\mathrm{I}_{1}(2 \sqrt{\alpha w})+\mathrm{J}_{1}(2 \sqrt{\alpha w})\right)$ \\
\hline 28 & $\frac{\left(8-\alpha^{2} t^{2}\right) \sin \alpha t-3 \alpha t \cos \alpha t}{8 \alpha^{5}}$ & $\frac{5 w}{8 \alpha^{4}} 2 F_{5}\left(\frac{1}{2}-i, \frac{1}{2}+i ; 1, \frac{3}{2}, \frac{3}{2},-\frac{1}{2}-i,-\frac{1}{2}+i ;-\frac{(\alpha w)^{2}}{16}\right)$ \\
\hline 29 & $\frac{t \sin \alpha t-\alpha t^{2} \cos \alpha t}{8 \alpha^{3}}$ & $\begin{aligned}-\frac{\sqrt{w}}{8 \alpha^{\frac{7}{2}}}( & \sqrt{2}\left(\operatorname{ber}_{1}(2 \sqrt{\alpha w})+\operatorname{bei}_{1}(2 \sqrt{\alpha w})\right) \\
& \left.+\sqrt{\alpha w} \operatorname{bei}_{0}(2 \sqrt{\alpha w})\right)\end{aligned}$ \\
\hline 30 & $\frac{3 t \sin \alpha t+\alpha t^{2} \cos \alpha t}{8 \alpha}$ & $\frac{w^{2}}{4}{ }_{1} F_{4}\left(3 ; \frac{3}{2}, \frac{3}{2}, 2,2 ;-\frac{(\alpha w)^{2}}{16}\right)$ \\
\hline 31 & $\frac{\left(3-\alpha^{2} t^{2}\right) \sin \alpha t+5 \alpha t \cos \alpha t}{8 \alpha}$ & $w_{1} F_{4}\left(3 ; 1,1, \frac{3}{2}, \frac{3}{2} ;-\frac{(\alpha w)^{2}}{16}\right)$ \\
\hline
\end{tabular}

Continued on next page 
Table 3 - Continued from previous page

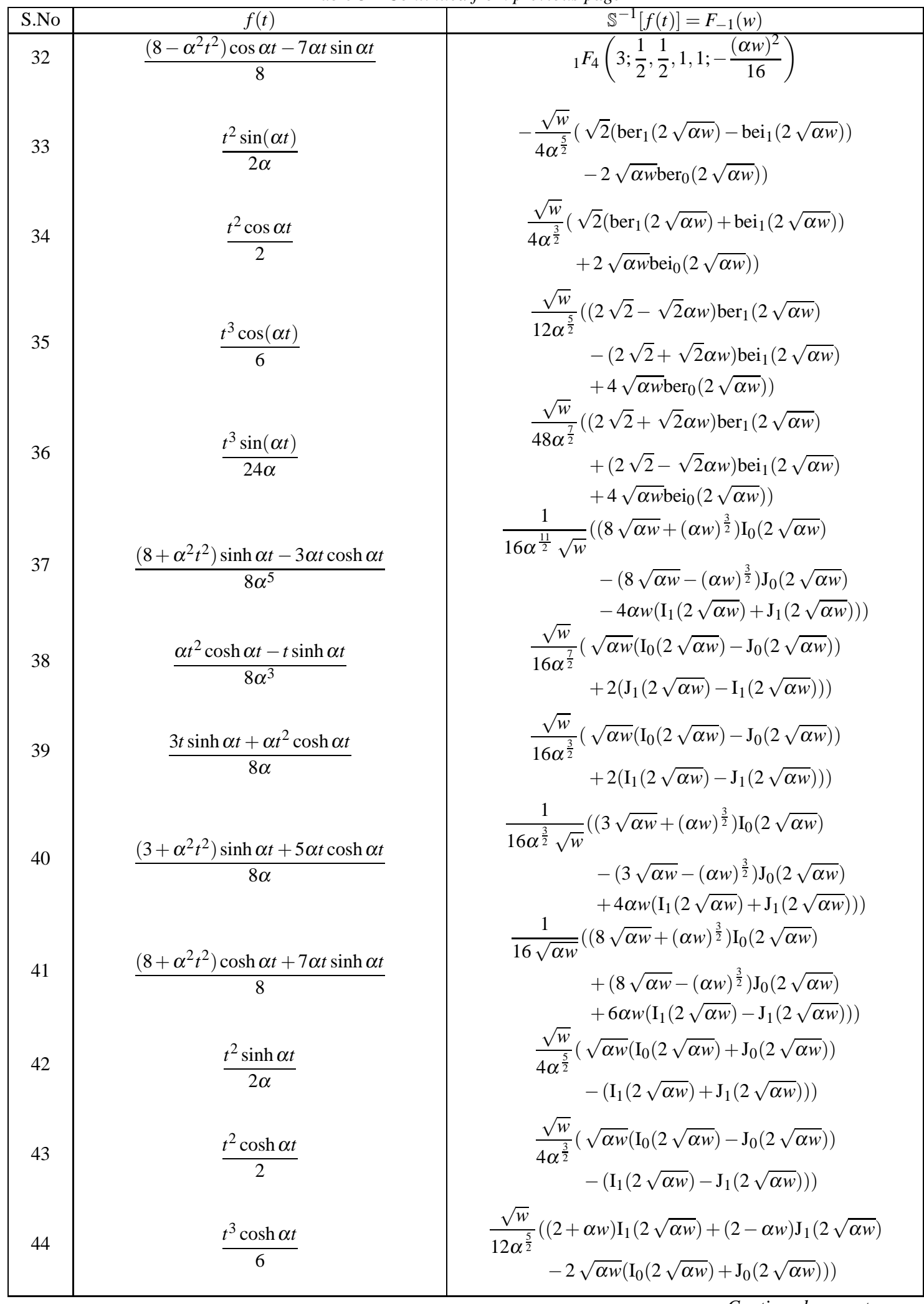


Table 3 - Continued from previous page

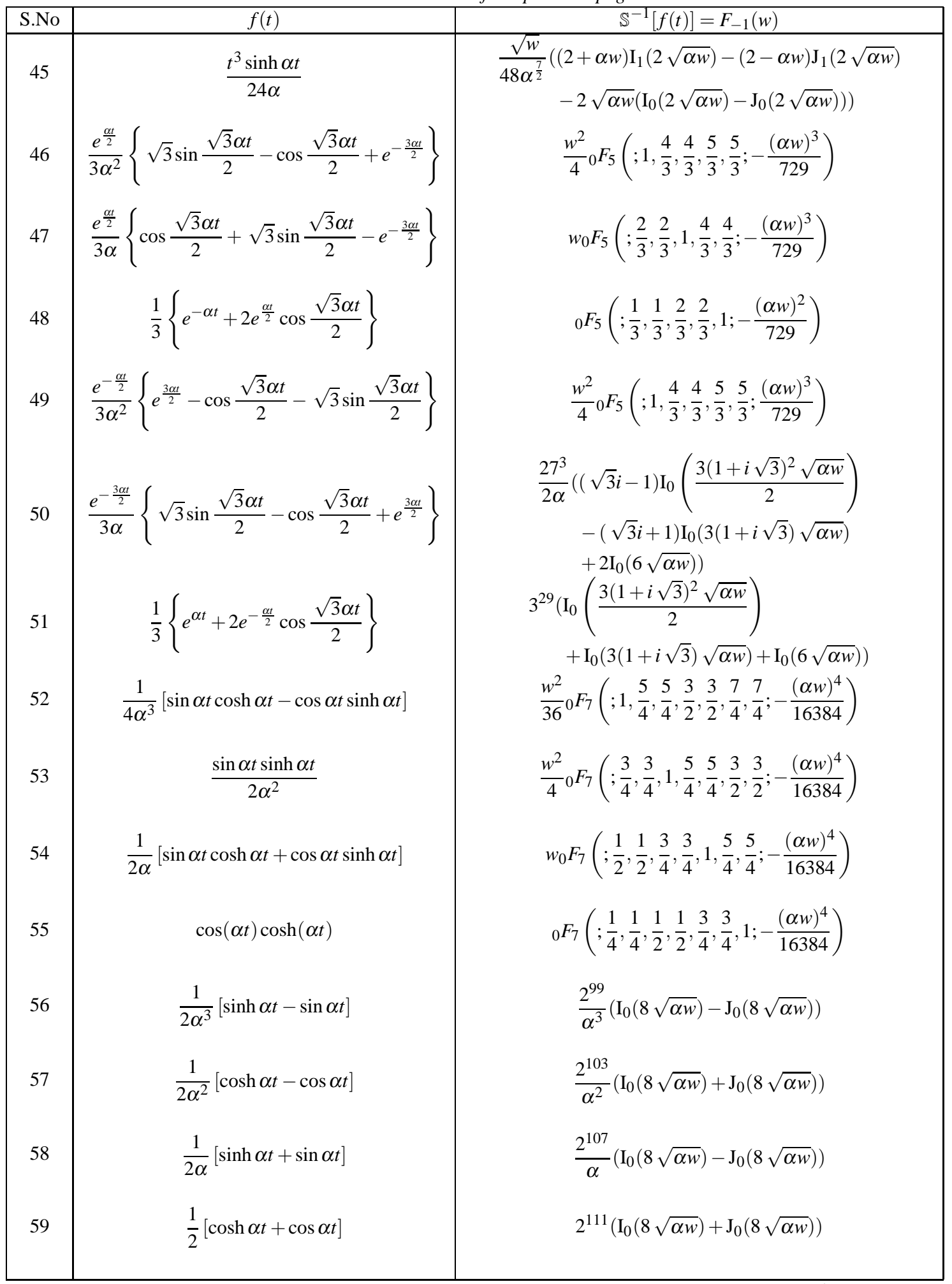

Continued on next page 
Table 3-Continued from previous page

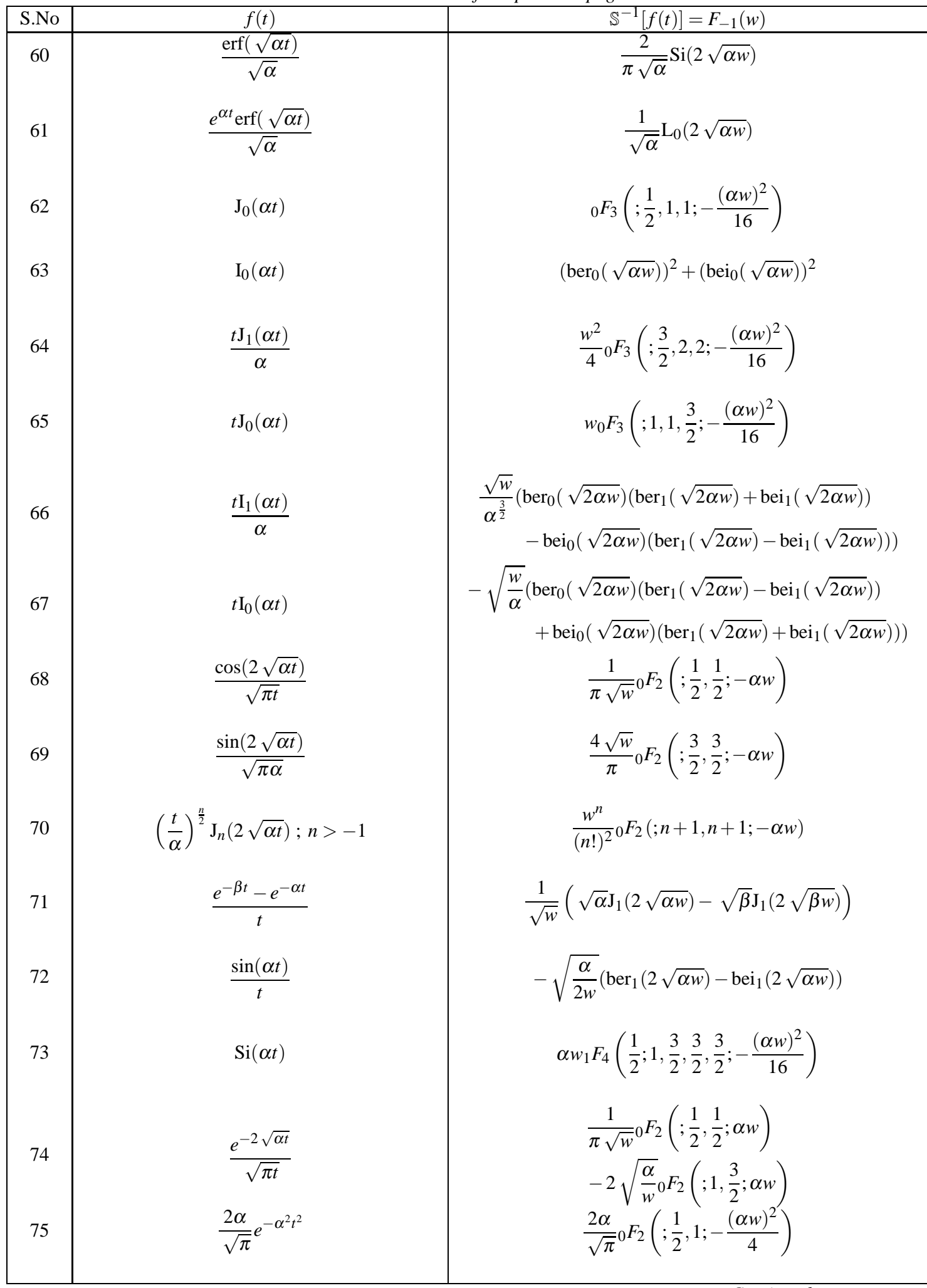

Continued on next page 
Table 3 - Continued from previous page

\begin{tabular}{|c|c|c|}
\hline S.No & $f(t)$ & $\mathbb{S}^{-1}[f(t)]=F_{-1}(w)$ \\
\hline 76 & $\operatorname{erf}(\alpha t)$ & $\frac{2 \alpha w}{\sqrt{\pi}}{ }_{1} F_{3}\left(\frac{1}{2} ; 1, \frac{3}{2}, \frac{3}{2} ;-\frac{(\alpha w)^{2}}{4}\right)$ \\
\hline 77 & $\frac{1}{\sqrt{\pi(t+\alpha)}}$ & $\frac{e^{-\frac{w}{2 \alpha}}}{\sqrt{\alpha \pi}} \mathrm{I}_{0}\left(\frac{w}{2 \alpha}\right)$ \\
\hline 78 & $\frac{1}{t+\alpha}$ & $\frac{1}{\alpha} e^{-\frac{w}{\alpha}}$ \\
\hline 79 & $\frac{1}{t^{2}+\alpha^{2}}$ & $\frac{1}{\alpha^{2}} \cos \left(\frac{w}{\alpha}\right)$ \\
\hline 80 & $\frac{t}{t^{2}+\alpha^{2}}$ & $\frac{1}{\alpha} \sin \left(\frac{w}{\alpha}\right)$ \\
\hline 81 & $\tan ^{-1}\left(\frac{t}{\alpha}\right)$ & $\operatorname{Si}\left(\frac{w}{\alpha}\right)$ \\
\hline 82 & $\frac{1}{2} \ln \left(\frac{t^{2}+\alpha^{2}}{\alpha^{2}}\right)$ & $\gamma+\ln \left(\frac{w}{\alpha}\right)-\operatorname{Ci}\left(\frac{w}{\alpha}\right) ; \gamma=0.5772156$ \\
\hline 83 & $\frac{1}{t} \ln \left(\frac{t^{2}+\alpha^{2}}{\alpha^{2}}\right)$ & $\frac{2}{w}\left(1-\cos \left(\frac{w}{\alpha}\right)\right)$ \\
\hline
\end{tabular}

Table 4: Special functions definition

\begin{tabular}{|c|c|c|}
\hline S.No & Function & Definition \\
\hline 1 & First kind Bessel function & $J_{n}(t)=\sum_{k=0}^{\infty} \frac{(-1)^{k}(t / 2)^{2 k+n}}{k !(n+k) !}$ \\
2 & Modified first kind Bessel function & $I_{n}(t)=\sum_{k=0}^{\infty} \frac{(t / 2)^{2 k+n}}{k !(n+k) !}$ \\
4 & Kelvin real function & $b e r_{n}(t)=\operatorname{Re} J_{n}\left(i^{\frac{3}{2}} t\right)$ \\
5 & Kelvin imaginary function & $b e i_{n}(t)=\operatorname{Im} J_{n}\left(i^{\frac{3}{2}} t\right)$ \\
6 & Guass hypergeometric series & ${ }^{2} F_{q}\left(\left(a_{p}\right) ;\left(b_{q}\right) ; t\right)=\sum_{k=0}^{\infty} \frac{\left(a_{1}\right)_{k} \cdot\left(a_{2}\right)_{k} \cdots\left(a_{p}\right)_{k} t^{k}}{\left(b_{1}\right)_{k} \cdot\left(b_{2}\right)_{k} \cdots\left(b_{q}\right)_{k} k !}$ \\
7 & Modified Struve function & $L_{v}(t)=\left(\frac{t}{2}\right)^{v+1} \sum_{k=0}^{\infty} \frac{(t / 2)^{2 k}}{\Gamma\left(k+\frac{3}{2}\right) \Gamma\left(k+v+\frac{3}{2}\right)}$ \\
& & $\operatorname{Sin}(t)=\int_{0}^{t} \frac{\sin (z) d z}{z}$ \\
\hline
\end{tabular}

Continued on next page 
Table 4 - Continued from previous page

\begin{tabular}{|c|c|c|}
\hline S.No & Function & Definition \\
\hline 8 & Cosine integral & $\operatorname{Ci}(t)=-\int_{t}^{\infty} \frac{\cos (z) d z}{z}$ \\
9 & Error function & $\operatorname{erf}(t)=\frac{2}{\sqrt{\pi}} \int_{0}^{t} e^{-z^{2}} d z$ \\
10 & Complementary error function & $\operatorname{erfc}(t)=\frac{2}{\sqrt{\pi}} \int_{t}^{\infty} e^{-z^{2}} d z$ \\
\hline
\end{tabular}

\section{Conclusion}

In this work an algorithm designed using inverse Sumudu transform DIST to solve differential equations and applied for verification of method to Whittaker and Zettl equations to obtain their new exact solutions. Graphical plots shows the complex plane behaviour of solutions for both equations. Inverse Sumudu transform of certain functions gives Bessel's, Kelvin related functions, Gauss hypergeometric series and modified Struve functions. Extensive list table of inverse Sumudu transform will be useful for further work.

\section{References}

[1] M. Abramowitz and I.A. Stegun, Handbook of mathematical functions, Dover Publications, Inc., New York, 1972.

[2] N.I. Akhiezer and I.M. Glazmann, Theory of linear operators in Hilbert space: I and II, Pitman and Scottish Academic Press, London and Edinburgh, 1981.

[3] D. Albayrak, S.D. Purohit, F. Ucar, On q-Analogues of Sumudu Transform, An. St. Univ. Ovidius Constanta, Vol. 21(1), 239âE"260, 2013

[4] W. O. Amrein, A. M. Hinz and D. B. Pearson, Sturm Liouville theory. Past and present. Birkhauser Verlag, Basel. 2005.

[5] F.B.M. Belgacem, Sumudu transform applications to Bessel's functions and equations. Appl. Math. Sci. 4(74), pp 3665-3686. 2010.

[6] F.B.M.Belgacem and R. Silambarasan, A distinctive Sumudu treatment of trigonometric functions. Journal. of Comput. Appl. Math. 312, pp 74-81. 2017.

[7] F.B.M. Belgacem, E.H. Al-Shemas and R. Silambarasan, Sumudu computation of the transient magnetic field in a lossy medium. Appl. Math. Inf. Sci. 11(1), pp 209-217. 2017.

[8] K. Jorgens, Spectral theory of second-order ordinary differential operators, Lecture Notes: Series no.2, Matematisk Institut, Aarhus Universitet, 1962/63.

[9] S. K. Panchal, Amol D. Khandagale, Pravinkumar V. Dole, Sumudu Transform of Hilfer-Prabhakar Fractional Derivatives and Applications, arXiv:1608.08017 [math.CA], 2016.

[10] M.R. Spiegel, Theory and Problems of Laplace Transforms, in: Schaums Outline Series, McGraw-Hill, New York, 1965.

[11] E.C. Titchmarsh, Eigenfunction expansions associated with second-order differential equations I, Clarendon Press, Oxford, 1962. 
[12] G.K. Watugala, Sumudu transform: a new integral transform to solve differential equations and control engineering problems, International Journal of Mathematical Education in Science and Technology, Volume 24, Issue 1, 1993.

[13] K. Wang, S. Liu, A new Sumudu transform iterative method for time-fractional Cauchy reactionâE "diffusion equation, Springerplus; 5(1): 865, 2016. 